\title{
Reactive Whole-Body Control: Dynamic Mobile Manipulation Using a Large Number of Actuated Degrees of Freedom
}

\author{
Alexander Dietrich, Thomas Wimböck, Alin Albu-Schäffer, and Gerd Hirzinger
}

\begin{abstract}
In this article, we present a control framework for reactive mobile manipulation of robotic systems with a large number of actuated degrees of freedom (DOF). We apply the concept to the humanoid robot Rollin' Justin of the German Aerospace Center (DLR). As service robotics is expected to be established in households and human environments in the near future, we consider relevant aspects like safety, compliance and robust task execution. The multi-DOF manipulator achieves an interactive redundancy resolution while planning algorithms only have to be applied to the low-dimensional operational space concerning task execution. Various experiments have been conducted, e.g., on reaching of a remote object, human-robot interaction, and self-collision avoidance of the manipulator. The results can serve as an interface to (re-)planning methods. Thanks to its interactivity, the approach can be applied in dynamic environments.
\end{abstract}

Index Terms-Mobile Manipulation, Redundancy, Force Control, Whole-Body Control, Impedance Control

\section{INTRODUCTION}

As a result of intensive research over the last decades, several robotic systems are approaching a level of maturity that allows robust task execution and safe interaction with humans and the environment. Particularly, when considering the aging of the population, service and household robotics is expected to play an important role in future domestic environments. In order to provide the ability to accomplish a huge range of tasks with different requirements, it appears to be inevitable to equip the robot with a large number of degrees of freedom (DOF). Just imagine an allegedly simple service task like filling a glass with water and placing it on a table. A variety of constraints has to be dealt with simultaneously: No liquid shall be slopped, collisions with the environment must be avoided and possible interactions with humans residing in the workspace of the robot have to be handled properly. And that is only a selection of objectives which indicates the necessity of a large number of DOF.

Another important topic concerns the motion characteristics of the manipulator. Who wants to have a service robot at home which behaves unpredictably compared to a human being? However, imitating human behavior and projecting it onto a robotic system is a big challenge [1].

But apart from appearance, versatility, and dexterity, another aspect is still more crucial: Safety, as Isaac Asimov stated in his 1st law in 1942: A robot may not injure a human being or, through inaction, allow a human being to come to harm. Beside applying sophisticated strategies to prevent dangerous situations in advance, the robot must also be capable

The authors are with the German Aerospace Center (DLR), Institute of Robotics and Mechatronics, Wessling D-82234, Germany;

Contact: alexander.dietrich@dlr.de

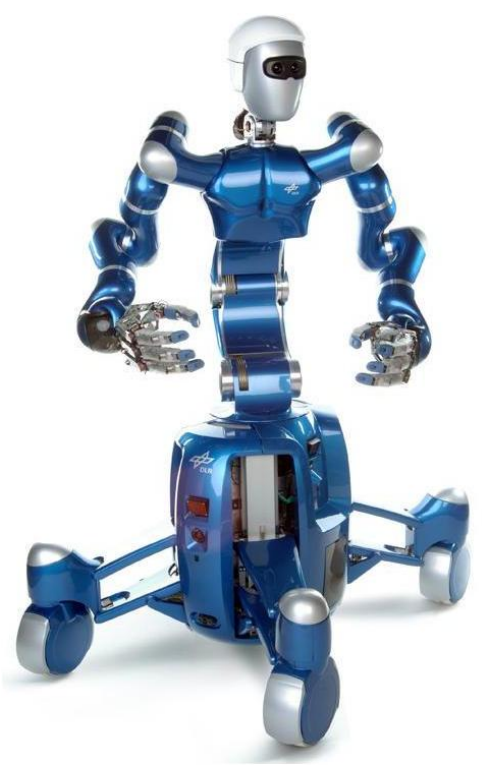

Fig. 1. Mobile humanoid Rollin' Justin of the German Aerospace Center (DLR) with 51 actuated degrees of freedom.

of feeling contact forces so as to react properly if a situation with physical human-robot interaction occurs [2].

Whether in movies or the press coverage, mainly humanoid robots are shown when robotic systems are addressed. From an engineering point of view, it is a big challenge to coordinate such a large number of degrees of freedom simultaneously. Beside humanoid robots like Honda's ASIMO [3], Robonaut 2 [4] and the HRP-2 robot [5], a variety of wheeled systems has been developed: Rollin' Justin [6], ARMAR-III [7], TWENDY-ONE [8], PR2 [9], to name just a few examples. But regardless of the specific structure of the system, the requirement of handling several objectives simultaneously is a common property. These range from features like precise task execution, collision avoidance and the compliance with physical constraints to higher level objectives as the realization of desired postures or maintaining the manipulability.

Based on the operational space formulation [10], many different methods have been developed for planning and reactive control of such systems [11], [12], [13]. In [14], multiple tasks are performed simultaneously on a biped humanoid robot in a whole-body control framework including issues like the control of the center of mass, obstacle avoidance and posture control. In [15], Brock and Khatib introduced the elastic strips framework that allows to execute previously planned motions in a dynamic environment. They reactively adapt to changes in the environment, e.g., when an obstacle is approaching the manipulator. The majority of these control strategies rests 
upon the design of artificial repulsive/attractive potential fields [16]. Having a large number of DOF, however, raises the question of a proper redundancy resolution. Especially when potential field-based strategies are applied, the problem of local minima in the case of competing objectives is crucial. An early technique by Siciliano and Slotine [17] utilizes the null space projection to derive joint velocities which execute a low priority task without disturbing any task with higher priority. Sentis and Khatib proceeded similarly in order to realize a hierarchy of behavioral primitives [11]. Another example for a consistent installation of a hierarchy can be found in [18], wherein a measure is imposed which indicates the feasibility of a task operating in the null space of a higher priority task. That coefficient may then lead to a transition changing the priority order in real-time. To integrate unilateral constraints into such a hierarchy, Mansard et al. proposed a control law based on a specific inverse operator so as to smooth the activation/deactivation process of subtasks [19].

As this article is about reactive, dynamic mobile manipulation, we have to define that term in the first place. In this context, reactive represents the ability to locally react on unpredictable, unmodeled dynamics and environments [16]. The word dynamic expresses the motion characteristics of the mobile manipulator. Motions are not executed slowly but they are fast enough such that dynamic effects have to be considered due to their significant influence. In the literature, mobile manipulation is mostly treated as a static problem to be solved in the high-dimensional configuration space [20]. Dynamic effects are taken into account quite scarcely [11], [14]. In this work, we incorporate the dynamics of the system. Moreover, we do not consider physical constraints on the planning level [21] but handle them reactively by utilizing the redundant DOF.

The article integrates the newest results of the robotic community on reactive, dynamic mobile manipulation control in a consistent framework, and gives solutions to several still open questions. The proposed framework allows to demonstrate the methodologies on a highly complex robotic system (see Fig. 1) with torque control interface at a high level of reliability and performance. The implementation in a $1 \mathrm{~ms}$ cycle comprises the simultaneous consideration of 9 reactive tasks which are integrated into a hierarchy with two basic levels. A further subdivision of these two levels is performed to specify the robot behavior in greater detail. Some of the tasks are highlighted in particular: A newly developed passivitybased algorithm for reactive avoidance of self-collisions [22] is presented and integrated into the whole-body control concept. Furthermore, dynamic singularities which describe a characteristic problem of non-holonomic platforms are dealt with by applying recently developed methods [23]. Experiments demonstrate the advantage of a variable footprint of the mobile base. Moreover, we focus on posture control and how to maintain the manipulability of the arms. Additionally, we give a short insight into a new concept for singularity-robust null space projections [24] which enhances the framework. In previous works, we have demonstrated the coordination between fingers and arms in terms of reactive two-handed manipulation. Here, the complete robot is controlled, utilizing all
TABLE I

ACTUATED DEGREES OF FREEDOM

\begin{tabular}{|c||c|c|}
\hline Subsystem & DOF & Control Mode \\
\hline \hline Torso & 3 & Torque \\
\hline Arms & $2 \times 7$ & Torque \\
\hline Hands & $2 \times 12$ & Torque \\
\hline Neck & 2 & Position/Velocity \\
\hline Platform \& Legs & 8 & Position/Velocity \\
\hline \hline$\sum$ & 51 & \\
\hline
\end{tabular}

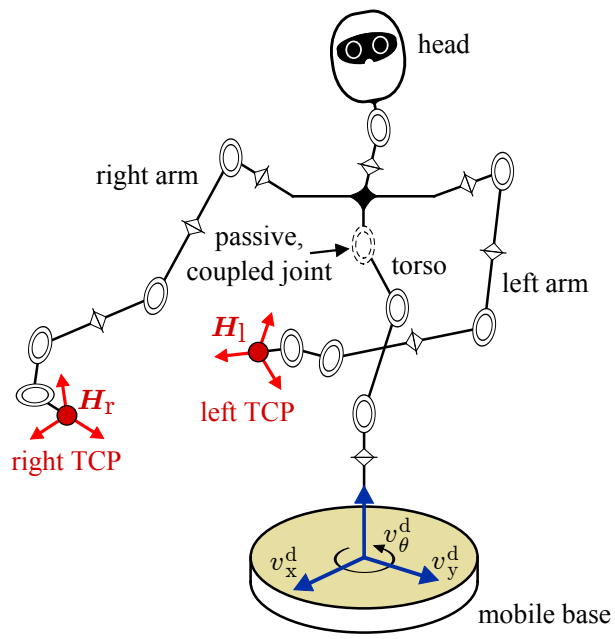

Fig. 2. Kinematic model of the robot illustrating the joints, the tool center points (TCP), the respective frames $\boldsymbol{H}_{\mathrm{r}}, \boldsymbol{H}_{1} \in S E(3)$, and the high level velocity interface $v_{\mathrm{x}}^{\mathrm{d}}, v_{\mathrm{y}}^{\mathrm{d}}$ and $v_{\theta}^{\mathrm{d}}$ [25] of the mobile base. The platform legs are not depicted.

51 DOF. The passivity-based whole-body control framework provides robust task execution which can be defined in the intuitive, low-dimensional Cartesian space. Hence, planning time can be saved significantly. Compared to admittance controlled systems, utilizing the torque interface allows compliant interaction with the environment and humans residing in the workspace of the robot. An extensive experimental section demonstrates the performance of our concept, validating compliant human-robot interaction, complex task execution and robot safety.

\section{SYSTEM OVERVIEW}

Our humanoid robot consists of an upper body system which is mounted on an omnidirectional, non-holonomic mobile base, see Fig. 1. The latter has a variable footprint which is realized by four extendable legs at whose ends the wheels are placed. The torque controlled upper body consisting of a torso, two arms, and two hands, is augmented by a head which is mounted on a position controlled pan-tilt unit. A stereo vision camera system is integrated in the head. The kinematic structure of the robot is illustrated in Fig. 2 and the total number of 51 actuated degrees of freedom is grouped by subsystem and control mode in Table I. 


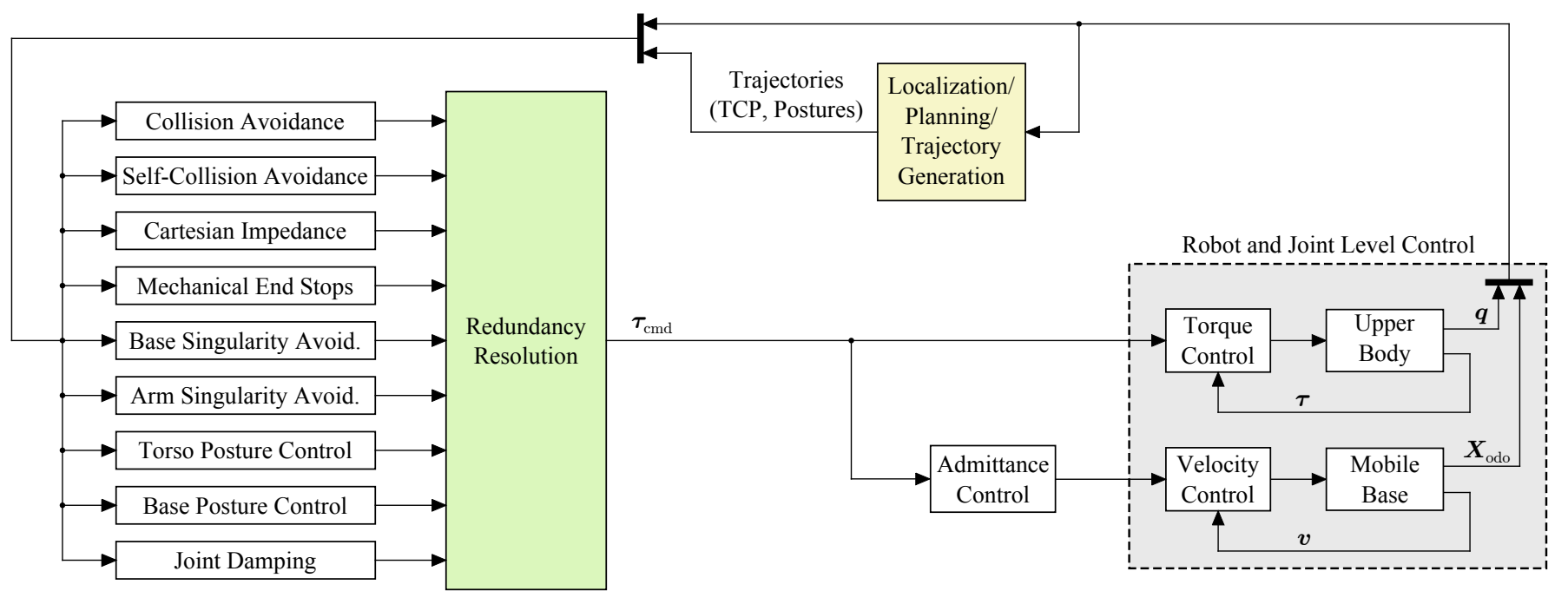

Fig. 3. Controller architecture for dynamic whole-body motions with 9 simultaneous tasks. The joint controllers of the robot (right) are fed by the redundancy resolution block (left) which gets input from the planning layer (center, top).

\section{CONTROL APPROACH}

This section starts with an overview of our controller architecture for dynamic whole-body mobile manipulation. Afterward, the basic components of that concept are explained in detail. First, the joint level controllers and their interfaces are presented. As ensuring safety is a crucial requirement in our framework, we continue with safety features before the treatment of physical constraints is specified. Subsequently, we give insight into our approach for robust task execution and various further subtasks as maintaining the manipulability of the arms or desired posture behaviors. The section ends with a short discussion on reactive control in general. Capabilities and limitations of reactivity are outlined.

\section{A. Overall Controller Architecture}

The schematic whole-body motion concept [26] is illustrated in Fig. 3. Fundamentally, one can divide the structure into three basic components. On the right, the robot model is shown. The torque controlled upper body and the velocity controlled mobile base provide measurements $\boldsymbol{q} \in \mathbb{R}^{43}$ and an estimation of the platform odometry $\boldsymbol{X}_{\text {odo }} \in \mathbb{R}^{3}$. An admittance coupling for the mobile base transforms the desired torques and forces into applicable commands for the kinematic velocity controller of the platform. At the top of Fig. 3, the high level logic is placed. In general, that includes the localization, planning algorithms, and the trajectory generation concerning the TCPs and desired postures of the robot. On the left, the redundancy resolution is illustrated for the generic case. Therein, a variety of 9 simultaneous tasks is considered which will be particularized in the remainder of this section.

\section{B. Design Choice of the Subtasks}

The natural question arises: Which criteria are relevant for the choice and prioritization of appropriate (sub-)tasks? At first glance, the selection in Fig. 3 (left side) may seem arbitrary. However, it follows some basic and intuitive rules that are essential for a proper robotic behavior. In this respect, we have drawn up four types of basic requirements that should be met:

1) safety,

2) physical constraints,

3) task execution,

4) posture primitives.

In our opinion, these four categories describe the key aspects. Considering such a guideline for the selection of the involved tasks is not a novelty but an intuitive basis of many well-known whole-body control approaches as [11].

Concerning the prioritization among these requirements, safety is usually located at the top end. By contrast, a posture primitive typically relates to a favored, though not essential task as effort minimization [14] or a desired posture [11]. Therefore, that aspect is suited as the lowest priority level and may be carried out if sufficient structural redundancy is left. The placement of the remaining two items in the list is more ambiguous. Although several physical constraints are crucial to prevent severe damage of the manipulator (e.g. avoidance of hitting joint limits), it might be reasonable to give higher priority to the task execution in some cases. That applies, for example, if the manipulator is sufficiently redundant w.r.t. the main task. Then the compliance with these physical constraints can be provided and the task execution does not have to be interfered by those tasks which are often defined by unilateral constraints. And that leads us directly to the second reason for an exchange of the physical constraints and the task execution within the hierarchy: The integration of unilateral, physical constraints into the higher levels of a task hierarchy causes additional problems in terms of discontinuities in the control law [19]. We will present a new solution to that problem for torque controlled robots in Sec. III-H.

Let us now return to the particular controller structure depicted in Fig. 3. The safety aspect is addressed by algorithms for collision avoidance with external objects and self-collision avoidance. A more detailed discussion on that topic will be given in Sec. III-D. The issue of physical constraints par- 
ticularly depends on the specific structure and characteristics of the considered system (Sec. III-E). In the case of Justin, physical limitations are reached in singular configurations of the mobile base. The design of a proper singularity avoidance is an appropriate remedy. Apart from that, the existence of mechanical end stops of the joints has to be taken into account. Task execution is realized by a Cartesian impedance, which is described in Sec. III-F. The last point in the list comprises additional posture behaviors or posture primitives [11], respectively. The structural redundancy of multi-DOF robots like Justin can be utilized to realize, for example, specific head poses, desired torso orientations, or arm postures. We restrict to torso and base postures as well as to non-singular arm configurations in Sec. III-G.

The total number of 9 tasks is a particular choice we have made here. Actually, the number, selection, and parameterization depends on many different aspects as the type of the main task, the structure of the environment, the desired dynamical behavior, and so forth. As an example, we recall the mentioned physical limitations of the mobile platform. They are only relevant for highly dynamic motions with fast rotations. In the case of slow tasks, they can be ignored. However, there are also indispensable tasks like the collision avoidance with external objects or the self-collision avoidance. As long as a reactive strategy is pursued, the danger of collisions must not be neglected. Besides, many physical constraints also have to be considered in any case in order to avoid damage of the manipulator itself. Think of a proper handling of mechanical end stops of joints as an example.

\section{Joint Level Control}

The basic control mechanisms of our humanoid can be divided into a dynamic and a kinematic part. On the one hand, the torque sensing in the upper body allows torque control. The respective framework introduced in [27] is wellestablished and validated by now. Therein, the torque feedback action is interpreted as a scaling of the apparent motor inertia. On the other hand, the mobile base is controlled in the kinematic domain via a dynamic feedback linearization [25]. As illustrated in Fig. 2 the algorithm makes it possible to apply velocity commands in the Cartesian directions instead of considering the alignment of wheel velocities and orientations separately. However, platform torque commands $\tau_{\mathrm{b}} \in \mathbb{R}^{3}$ cannot be applied unmodified. In this context, we utilize an admittance coupling:

$$
\boldsymbol{M}_{\mathrm{b}} \dot{\boldsymbol{v}}^{\mathrm{d}}+\boldsymbol{D}_{\mathrm{b}} \boldsymbol{v}^{\mathrm{d}}=\boldsymbol{\tau}_{\mathrm{b}}
$$

The desired velocities that are input of the kinematic controller are expressed by $\boldsymbol{v}^{\mathrm{d}}=\left(v_{\mathrm{x}}^{\mathrm{d}} v_{\mathrm{y}}^{\mathrm{d}} v_{\theta}^{\mathrm{d}}\right)^{T}$. Applying such an admittance allows to set a virtual platform inertia $\boldsymbol{M}_{\mathrm{b}}$. Analogously, damping can be injected via $\boldsymbol{D}_{\mathrm{b}}$. The access to the neck joints is related to this approach but will be specified in a later section.

\section{Ensuring Robot Safety}

In our approach, the aspect of safety is taken into account by three separate technologies:
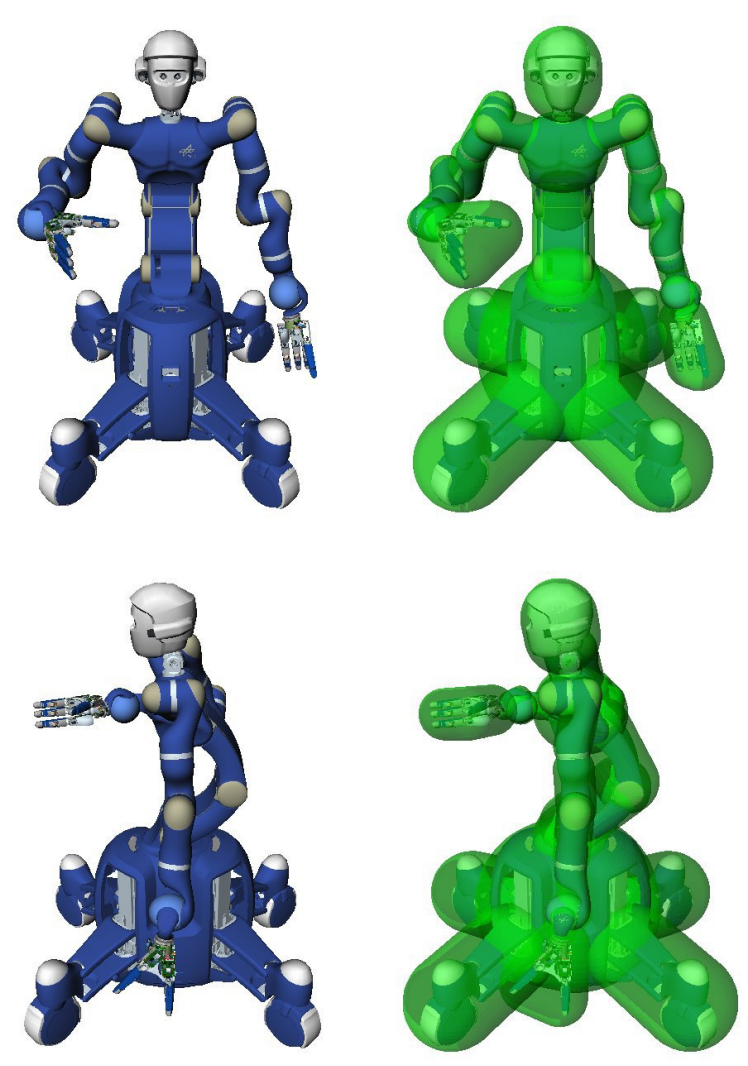

Fig. 4. Collision model consisting of 28 hulls (left arm: 8 , right arm: 8 , mobile base: 5 , torso: 4 , head: 2 , floor: 1 )

- Torque sensing in the upper body joints facilitates compliant behavior which allows safe physical human-robot interaction that avoids clamping situations due to collision detection [2].

- As the mobile base is velocity controlled and not equipped with force/torque sensors, hitting an object would not be recognized. For this reason, four timeof-flight cameras are integrated into the platform frame. Based on these, artificial repulsive potential fields can be designed to repel the platform from detected objects.

- The large number of DOF of Rollin' Justin requires appropriate handling of self-collision scenarios. A passive, reactive, potential field-based algorithm [22] has been developed which we will present in the following:

Initially, we determine potentially colliding body parts and generate repulsive forces between them in the second step. To this end, we have established a geometrical model which consists of spherically extended convex hulls placed around the robot body links, see Fig. 4 (right). Based on that virtual model, an efficient distance computation algorithm [28] has been adapted that outputs $n_{\mathrm{p}}$ point pairs on potentially colliding body links in real-time (sampling time: $1 \mathrm{~ms}$ ). Finally, these pairs are used to generate the repulsive forces. 


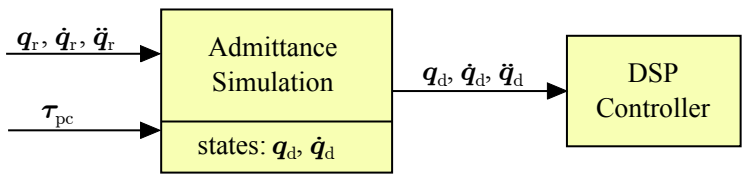

Fig. 5. Admittance simulation to incorporate position controlled joints (subscript pc) into a force/torque control framework. The reference input $\boldsymbol{q}_{\mathrm{r}}$ is deviated and the desired joint configuration $\boldsymbol{q}_{\mathrm{d}}$ results.

Feasible self-collision avoidance torques $\tau_{\text {coll }}$ can be derived by

$$
\boldsymbol{\tau}_{\mathrm{coll}}=-\left(\frac{\partial V_{\mathrm{coll}}(\boldsymbol{q})}{\partial \boldsymbol{q}}\right)^{T}-\boldsymbol{D}_{\mathrm{coll}}(\boldsymbol{q}) \dot{\boldsymbol{q}}
$$

wherein $V_{\text {coll }}(\boldsymbol{q})$ describes a repulsive potential field sited on the surface of the collision model with respect to the joint configuration $\boldsymbol{q}$. Damping is injected by the positive semi-definite damping matrix $\boldsymbol{D}_{\text {coll }}(\boldsymbol{q})$. For its evaluation, we consider the actual inertia distribution $\boldsymbol{M}(\boldsymbol{q})$ :

$$
\boldsymbol{D}_{\text {coll }}(\boldsymbol{q})=\mathcal{D}\left(\boldsymbol{M}(\boldsymbol{q}), \boldsymbol{K}_{\text {coll }}(\boldsymbol{q}), \boldsymbol{\zeta}\right)
$$

In (3), $\boldsymbol{K}_{\text {coll }}(\boldsymbol{q})$ describes the actual, virtual potential stiffness. As damping method $\mathcal{D}$, we apply the double diagonalization approach [29] in order to realize desired damping ratios $\zeta$. The elements of $\zeta$ relate to the $n_{\mathrm{p}}$ contact point pairs and specify the damping behavior in the respective collision direction.

If subsystems without force/torque sensing are represented in the collision model, the respective self-collision avoidance torques have to be transformed into proper joint trajectories for the position controlled subsystems. Considering our robot, such an admittance interface has to be utilized for the two neck joints as well as for the mobile platform (1). Fig. 5 illustrates the approach schematically. The torque $\tau_{\mathrm{pc}}$ deviates a reference joint trajectory $\boldsymbol{q}_{\mathrm{r}}$ and results in $\boldsymbol{q}_{\mathrm{d}}$ which can be directly applied. An easy realization of the admittance simulation is a linear mass-spring-damper relation.

In experiments [22] it turned out that the choice of a configuration dependent damping is an effective means to specifically dissipate kinetic energy which is stored within moving body links. Fig. 6 (left) shows the initial configuration during such an experiment. The robot is controlled in gravity compensation mode ${ }^{1}$ and the user throws the left forearm onto the right one, see Fig. 6 (right). We repeated the experiment for different damping parameterizations which range from zero damping $(\zeta=0)$ to an overdamped system with $\zeta=1.3$. Fig. 7 takes account of the most critical body pairs which are "Left Hand \& Right Hand" and "Left Hand \& Right Wrist". The upper plots show the distance between the involved links. Apparently, a higher damping leads to a significantly slower motion when the links are diverging again. After penetrating the potential fields at a distance of $d_{0}=0.15 \mathrm{~m}$, repulsive forces are generated which are depicted in the second row diagrams. Consistently with the penetration of the potential fields in the upper plots, a higher damping requires lower repulsive forces in general. Finally, the bottom graphs show

\footnotetext{
${ }^{1}$ Static gravity compensation is achieved by utilizing the torque control interface and a configuration dependent model of the inertia distribution of the manipulator.
}

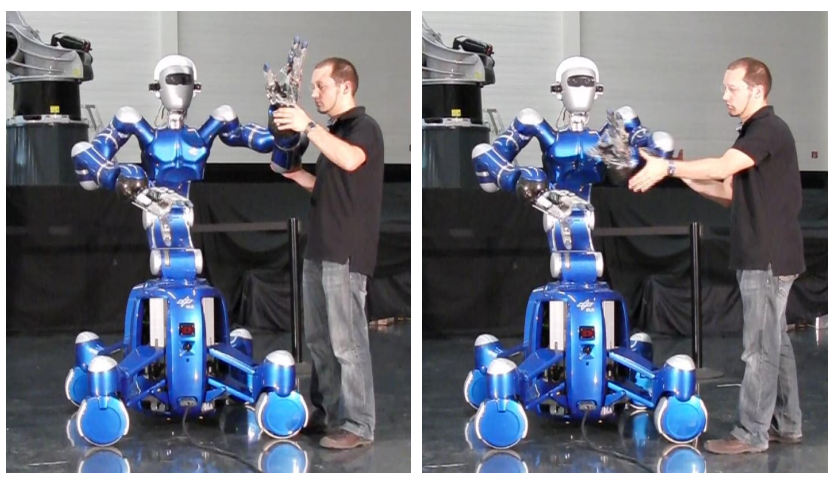

Fig. 6. Starting position (left) and snapshot during the experiment on selfcollision avoidance (right).

the computed damping forces. The fact that they are not continuously differentiable is due to the choice of the potentials as $C^{2}$ functions. The $C^{0}$ damping force directly depends on the stiffness, i.e., the second derivative of the potential function. Beside the depicted contact point pairs in Fig. 7, several additional repulsions between potentially colliding links emerge during the experiment. In the considered case, a total number of 14 pairs is involved.

\section{E. Complying with Physical Constraints}

Depending on the mechanical structure of the robot, various issues concerning physical limitations have to be handled appropriately. Probably the most common one refers to mechanical end stops of joints. A well-known method to prevent impacts on the drives is to avoid regions around the mechanical limit stops. Mostly, artificial potential fields are designed to repel from those undesired configurations. In order not to constrict the working range of the robot, these fields are supposed to be unilateral and as small as possible. However, integrating unilateral constraints into a task hierarchy requires proper handling of the activation/deactivation process. In this respect, a new concept has been developed which is explained in Sec. III-H.

Apart from that, another limitation has to be taken into account when considering a wheeled mobile manipulator like Rollin' Justin. In general, arbitrary translational and rotational trajectories of the platform may be realized. However, dynamic singularities exist which lead to infeasible control inputs and hence to stressing of the mechanical structure. A consistent motion of the platform can only be achieved if the wheels are aligned to the instantaneous center of rotation (ICR) which is defined by the translational and rotational velocity of the base:

$$
\left(\begin{array}{l}
x_{\mathrm{ICR}} \\
y_{\mathrm{ICR}}
\end{array}\right)=\mathcal{F}\left(v_{\mathrm{x}}, v_{\mathrm{y}}, v_{\theta}\right)
$$

The non-injective function $\mathcal{F}$ describes the relation between the Cartesian velocities of the mobile base and the location of the ICR which is defined by the coordinates $x_{\mathrm{ICR}}$ and $y_{\mathrm{ICR}}$. A graphical interpretation of (4) is given in Fig. 8. The wheel orientations and velocities $v_{\mathrm{w}, 1}$ to $v_{\mathrm{w}, 4}$ align to the ICR.

If the ICR approaches one wheel, its steering velocity increases to fulfill the hard kinematic constraint. Crossing a 

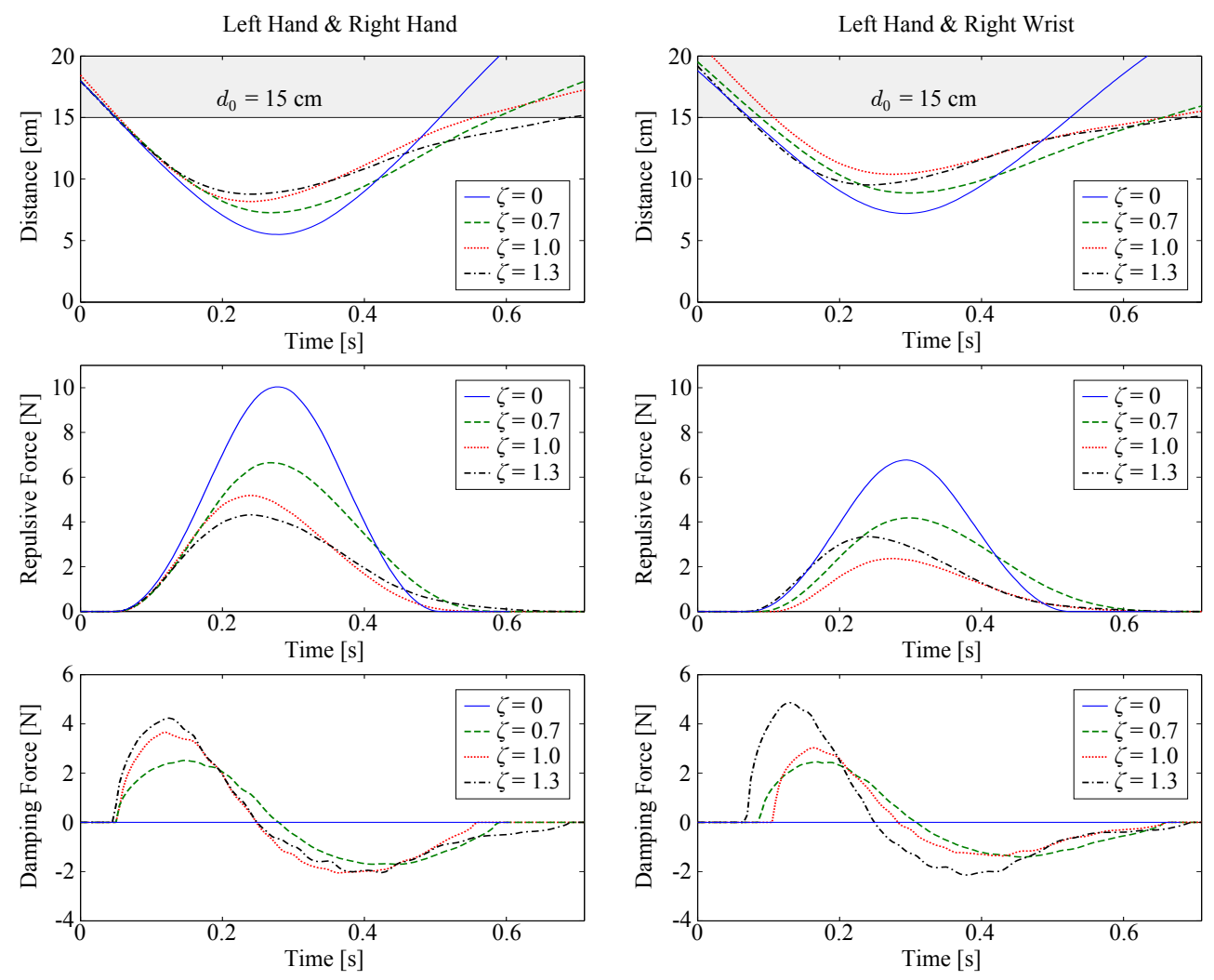

Fig. 7. Self-collision avoidance between left hand and right hand/wrist. The distances between the potentially colliding links (upper plots) generate repulsive forces (center plots). The velocities of the approaching body parts lead to configuration dependent damping forces (bottom plots).

wheel center point would require an infinite steering velocity which is obviously not feasible. Therefore, these dynamic configurations have to be avoided. Beside applying constraints on the accessible velocity space [30], also a reactive method [31] has been introduced to tackle the problem. In [23], we proposed such an approach which is again based upon repulsive potential fields. The ICR is actively repelled from the wheels so as to keep the required steering velocities bounded. The placement of the fields is illustrated in Fig. 9. A peculiarity of mobile platforms we can benefit from is that through the accelerations in $\mathrm{x}-, \mathrm{y}$ - and $\theta$-direction, we may directly affect the ICR velocity. Therefore, we are able to abruptly stop the motion of the ICR without deceleration, if necessary. This is due to the fact that the ICR is just a virtual point and does not possess an inherent inertia.

As a peculiarity of the variable footprint of the platform, we are also able generate repulsive forces acting independently on each wheel in leg-direction in order to repel the wheels from the ICR. Hence, a two-sided repulsion is achieved to avoid the singular configurations. Adaptable potential fields have to be used in order to prevent overlaps which would result in obstructive local minima. The strategy is demonstrated in Fig. 10. Notice that the definition of a single ICR for the whole platform does not hold any longer in the case of such leg maneuvers, since each wheel becomes a separate rigid body while moving.

We present results from simulations and experiments on the real system in Fig. 11. In this experiment, the leg lengths are kept constant. A trajectory starting at time $t_{\text {start }}$ is depicted in the ICR space and has been validated in simulation (with and without repulsion of the ICR) and experiments (activated ICR repulsion). Critical situations occur at time $9 \mathrm{~s}, 15 \mathrm{~s}$, and $18 \mathrm{~s}$. In the case of activated control, the ICR path is deviated. The corresponding steering velocities are plotted in Fig. 12. Evidently, the peaks from the non-controlled case are lowered when ICR repulsion is active. However, shifting the ICR has to be paid with a deviation from the nominal trajectory. Thus a trade-off between steering velocity and tracking performance has to be found.

In the following experiment, the additional repulsion of the wheels is activated. Such a leg maneuver does not have to be paid with anything but the varying footprint. A demonstration is provided by Fig. 13, wherein the wheel center point moves from $\mathbf{A}$ to $\mathbf{B}$. In this example, the deviations from the nominal trajectory are $32 \%$ below the values of locked legs. A more detailed discussion on all these experimental results can be found in [23].

\section{F. Task Execution}

As a benefit of the autonomous whole-body coordination of our highly redundant robot, a task can be defined in the lowdimensional Cartesian space of the TCPs. We implemented a passive impedance control [32] which complies with the law

$$
\boldsymbol{\tau}_{\text {imp }}=\boldsymbol{g}(\boldsymbol{q})-\left(\frac{\partial V_{\mathrm{imp}}\left(\boldsymbol{q}, \boldsymbol{X}_{\mathrm{odo}}, t\right)}{\partial \boldsymbol{q}}\right)^{T}-\boldsymbol{D}_{\mathrm{imp}}(\boldsymbol{q}) \dot{\boldsymbol{q}} .
$$

Gravity effects are compensated by $\boldsymbol{g}(\boldsymbol{q})$. A spatial spring which is spanned between actual and desired TCP frame is 
defined by the potential $V_{\text {imp }}\left(\boldsymbol{q}, \boldsymbol{X}_{\text {odo }}, t\right)$. Due to the mobility of the manipulator, the odometry $\boldsymbol{X}_{\text {odo }}$ is required here. Additional damping is injected through $\boldsymbol{D}_{\text {imp }}(\boldsymbol{q})$. From a passivity point of view, any positive semi-definite matrix can be applied. Within this work, $\boldsymbol{D}_{\mathrm{imp}}(\boldsymbol{q})$ is chosen such that desired damping ratios are realized. This is again achieved by utilizing the double diagonalization [29] of the effective mass matrix and the Hessian of the potential function, i.e., $\partial^{2} V_{\text {imp }}\left(\boldsymbol{q}, \boldsymbol{X}_{\text {odo }}, t\right) / \partial \boldsymbol{q}^{2}$, in task coordinates.

The potential according to the spatial spring which is spanned between two frames $\boldsymbol{H}_{1} \in S E(3)$ and $\boldsymbol{H}_{2} \in S E(3)$ will be denoted by $V_{\mathrm{s}}\left(\boldsymbol{H}_{1}, \boldsymbol{H}_{2}, \mathcal{K}\right)$, wherein $\mathcal{K}$ represents the parameterization of the impedance, i. e., the spatial stiffness. Correspondingly, $V_{\mathrm{imp}}\left(\boldsymbol{q}, \boldsymbol{X}_{\text {odo }}, t\right)$ can be expressed by

$$
\begin{aligned}
V_{\text {imp }}\left(\boldsymbol{q}, \boldsymbol{X}_{\text {odo }}, t\right)= & V_{\mathrm{s}}\left(\boldsymbol{H}_{\mathrm{r}}\left(\boldsymbol{q}, \boldsymbol{X}_{\text {odo }}\right), \boldsymbol{H}_{\mathrm{r}}^{\mathrm{d}}(t), \mathcal{K}_{\mathrm{r}}\right)+ \\
& V_{\mathrm{s}}\left(\boldsymbol{H}_{\mathrm{l}}\left(\boldsymbol{q}, \boldsymbol{X}_{\text {odo }}\right), \boldsymbol{H}_{\mathrm{l}}^{\mathrm{d}}(t), \mathcal{K}_{\mathrm{l}}\right)
\end{aligned}
$$

since both the right and the left TCP are regarded (subscripts $\mathrm{r}$ and $\mathrm{l})$.

\section{G. Maintaining the Manipulability and Realizing Desired Impedances for Torso and Base}

The structural redundancy of the robot can be utilized in order to maintain a proper manipulability. In this respect, a singularity avoidance for the arms has been designed to prevent rank deficiency of the Jacobian matrix $\boldsymbol{J}_{\mathrm{C}}(\boldsymbol{q})$ of the Cartesian impedance. A spatially bounded potential function is set up to keep the kinematic manipulability measure

$$
m_{\text {kin }}(\boldsymbol{q})=\sqrt{\operatorname{det}\left(\boldsymbol{J}_{\mathrm{C}}(\boldsymbol{q}) \boldsymbol{J}_{\mathrm{C}}(\boldsymbol{q})^{T}\right)}
$$

on a sufficiently high level. More details on the algorithm by Ott can be found in [32].

In terms of providing an unrestricted stereo vision, specific postures of the torso support the neck joint actuators in positioning the camera system in the head. Likewise, a separate trajectory for the mobile base enables to handle obstacles in the workspace of the robot which are known in advance or

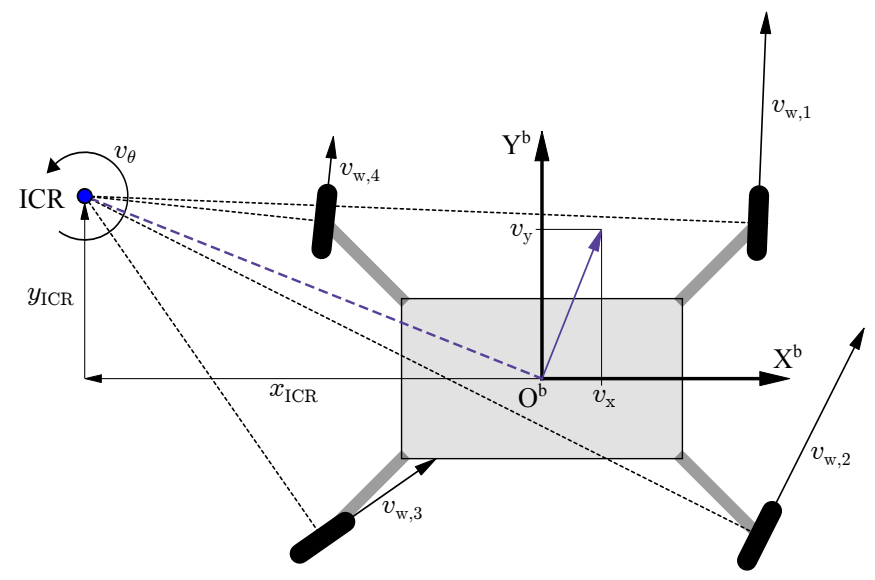

Fig. 8. Consistent motion of the mobile platform around the instantaneous center of rotation (ICR), depicted in the platform body frame (superscript b). The whole structure is one rigid body since the leg lengths are kept constant here.

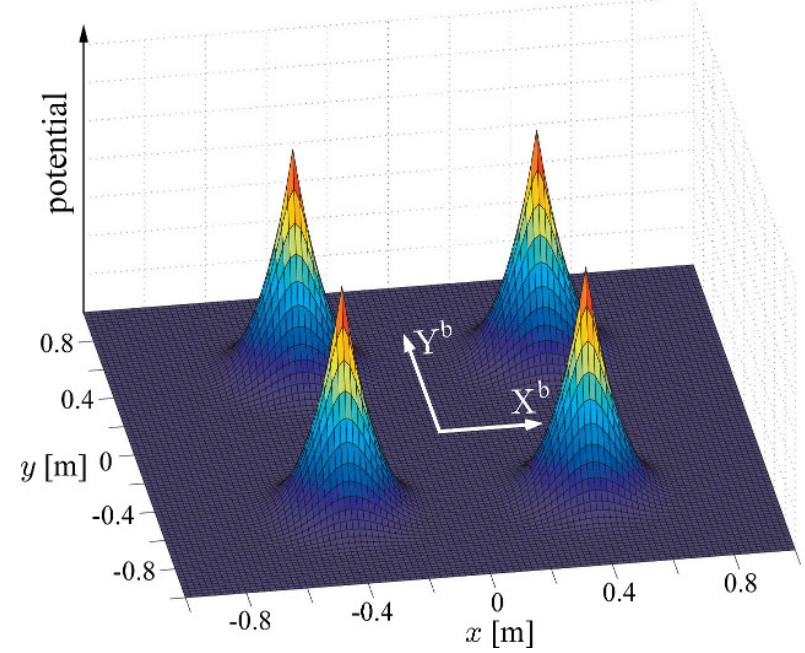

Fig. 9. Repulsive potentials placed around the wheel center points to repel the ICR from the wheels.

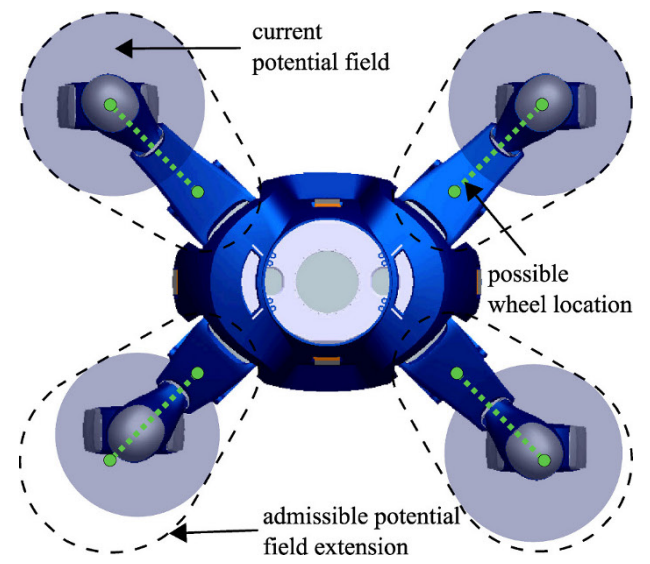

Fig. 10. Feasible potential field extensions for the ICR repulsion. The extension depends on the location of the wheels to prevent obstructive overlaps.

given by a higher level planning instance online. A potential function can be set up including these two issues (subscript $t$ for torso and $b$ for mobile base):

$$
\begin{aligned}
V_{\mathrm{tb}, \text { imp }}\left(\boldsymbol{q}, t, \boldsymbol{X}_{\text {odo }}\right)= & V_{\mathrm{s}}\left(\boldsymbol{H}_{\mathrm{t}}(\boldsymbol{q}), \boldsymbol{H}_{\mathrm{t}}^{\mathrm{d}}(t), \mathcal{K}_{\mathrm{t}}\right)+ \\
& V_{\mathrm{s}}\left(\boldsymbol{H}_{\mathrm{b}}\left(\boldsymbol{X}_{\text {odo }}\right), \boldsymbol{H}_{\mathrm{b}}^{\mathrm{d}}(t), \mathcal{K}_{\mathrm{b}}\right)
\end{aligned}
$$

The parameterizations of the impedances to realize the postures are specified by $\mathcal{K}_{\mathrm{t}}$ and $\mathcal{K}_{\mathrm{b}}$.

\section{H. Redundancy Resolution to Deal with Singular Jacobian Matrices and to Integrate Unilateral Constraints into the Task Hierarchy}

We are currently working on a novel technique to deal with singular Jacobian matrices concerning their null space projectors [24]. The method can also be utilized to integrate unilateral constraints into a task hierarchy [33], for example, repulsive potentials with limited potential extension. As long as they are deactivated, the projection into the null space of that task shall be unrestricted, i. e., described by the identity matrix $\boldsymbol{I}$. The activation locks certain directions abruptly due 


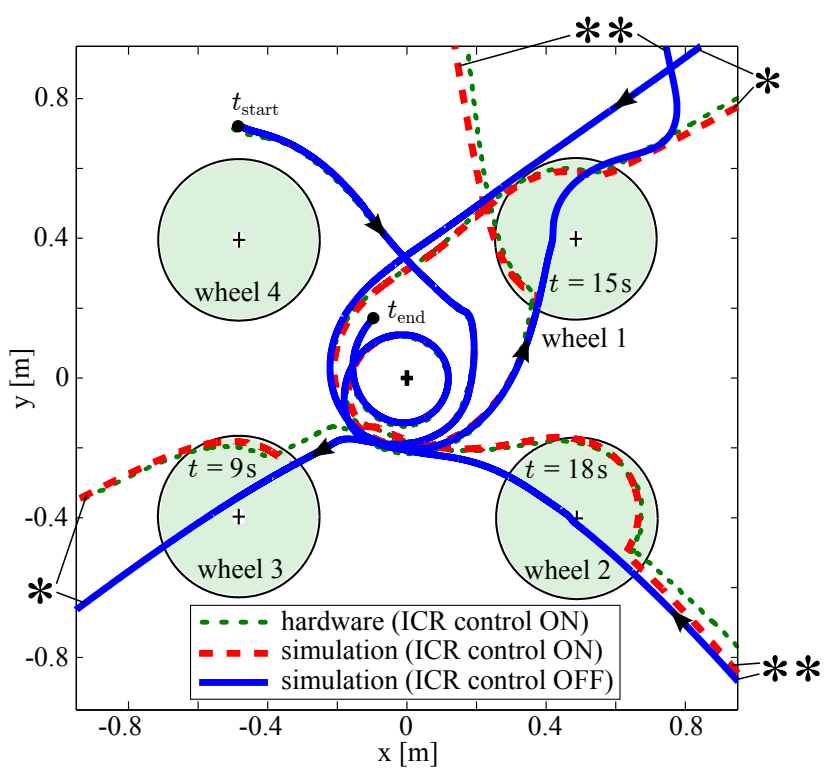

Fig. 11. The instantaneous center of rotation in the platform body frame. Activated ICR control avoids the singular locations around the wheel center points.
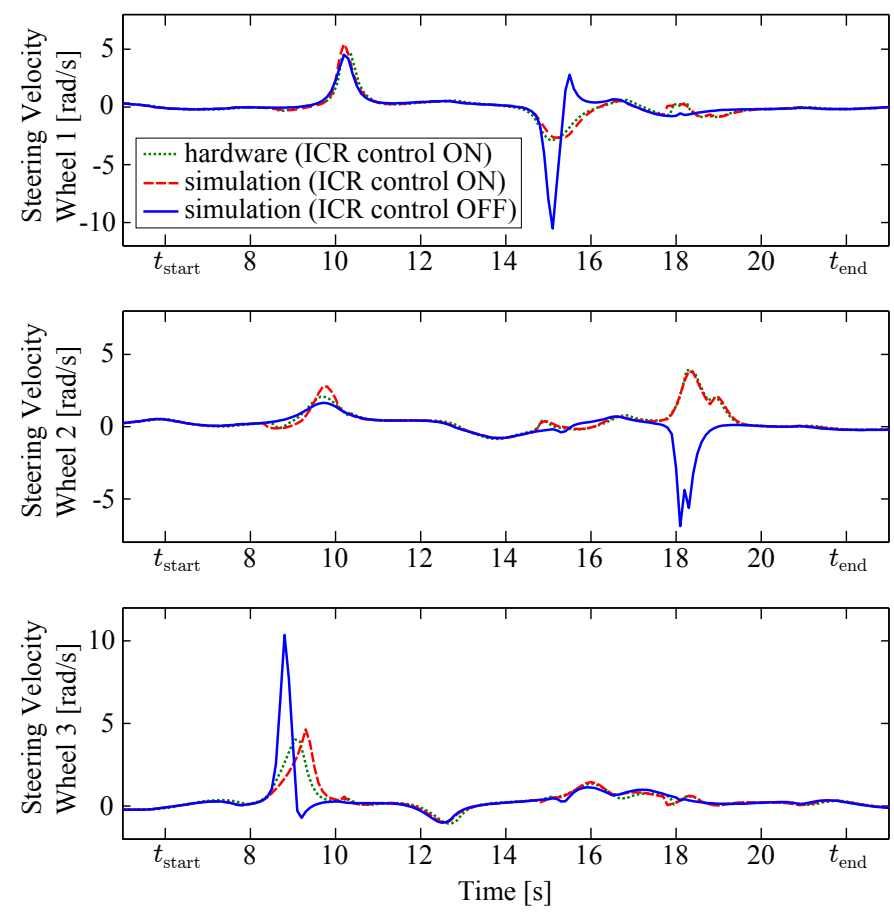

Fig. 12. Steering velocities of wheels 1,2 , and 3 for activated and deactivated ICR repulsion in simulation and experiment. The peaks directly correspond to the arising singularities indicated in Fig. 11.

to a change of the rank of the Jacobian matrix $\boldsymbol{J} \in \mathbb{R}^{m \times n}$ of the primary task ( $n$ : number of DOF; $m$ : dimension of task). By applying conventional approaches, that activation process induces discontinuities in the control law. In this context, we developed a new method to provide a controlled, smooth transition online by specifically limiting the torque derivative which results from the projection via the null space projector $N$.

$$
\boldsymbol{N}=\boldsymbol{I}-\boldsymbol{V} \boldsymbol{A}_{\mathrm{des}} \boldsymbol{V}^{T}
$$

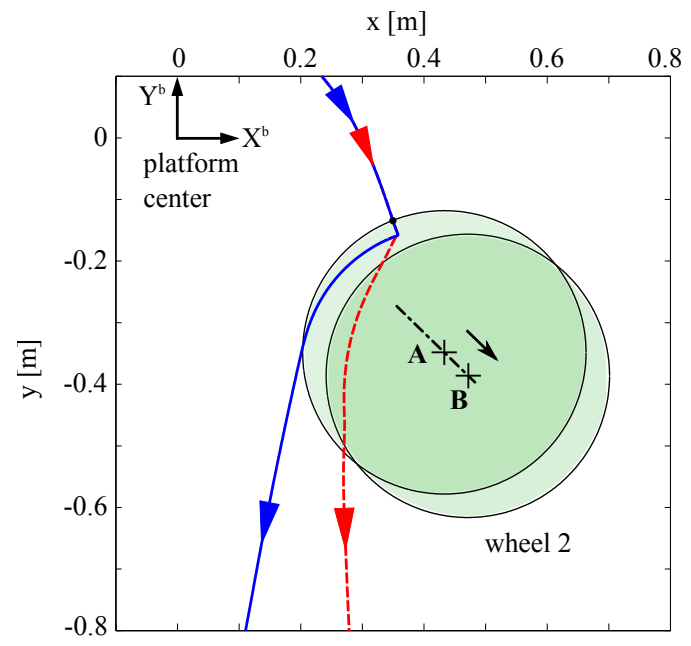

Fig. 13. Simulated ICR in body frame (blue/solid: deactivated leg motion, red/dashed: activated leg motion, black/chain dotted: possible wheel location).

$$
\boldsymbol{A}_{\mathrm{des}}=\operatorname{diag}\left(a_{\mathrm{des}, 1}, a_{\mathrm{des}, 2}, \ldots, a_{\mathrm{des}, m}, \mathbf{0}_{1 \times(n-m)}\right)
$$

The right-singular vectors or the directions of the Jacobian matrix, respectively, are comprised by $\boldsymbol{V}$. Through the variable diagonal activator matrix $\boldsymbol{A}_{\mathrm{des}}$, we are able to smooth the activation/deactivation process of the respective directions. By shaping the elements $a_{\mathrm{des}, 1}$ to $a_{\mathrm{des}, m}$ continuously within the range $0 \ldots 1$, the transition is smoothed. Frequently, unilateral constraints are described by row vector Jacobians. Just think of the mechanical end stops (Sec. III-E) or the arm singularity avoidance (Sec. III-G), to name just two examples. In such a case, (9) degrades to a simple multiplication including the normalized Jacobian row vector and the first element of $\boldsymbol{A}_{\mathrm{des}}$. No singular value decomposition or any matrix inversion has to be performed which turns the method into a very computing time efficient technique within the redundancy resolution concept. Furthermore, the method is particularly intuitive due to the geometrical interpretation.

\section{Discussion on Reactive Control - Capabilities and Limita- tions}

The major advantage of reactive methods is the capability of dealing with unforeseen events, unmodeled environments and the unpredictability of, for example, human behavior in the workspace of the robot. Nevertheless, reactivity has the wellknown drawback of being only a local method that always suffers from local minima and the lack of global information about the scene. There is no way around a global planning level. Indeed, utilizing reactive components may reduce the high level path planning burden [13], as Khatib has already stated in 1986 [16], but only the combination with a planning layer is able to resolve those issues. If the intensity of a disturbance is too high, online replanning becomes necessary.

Although the article does not particularly focus on planning, we want to draw attention to this very essential issue. In Fig. 3, an indication has already been given by the block "Localization/Planning/Trajectory Generation" in the signal flow chart. A wide field of research focuses on online path planning or trajectory modification subject to real-time sensor 
data [34], [35]. Key issues are fast trajectory computation in order to allow real-time applicability as well as a smart usage of global data.

\section{EXPERIMENTAL VALIDATION OF THE WHOLE-BODY CONTROL}

In the following, the redundancy resolution from [26] is utilized and experiments are performed. The hierarchy is specified as follows:

- The top priority is defined by a superposition of the safety features (collision avoidance, self-collision avoidance) and the task execution (Cartesian impedance w.r.t. the TCPs). The latter is designed to be outplayed by the safety tasks if necessary.

- The secondary tasks (singularity avoidances, mechanical end stops, impedances for torso and base, null space damping) are realized in the null space of the Cartesian impedance.

As explained in Sec. III-B, some physical constraints are given a lower priority than the task execution because a large number of DOF is available on Justin in order to comply with these constraints without disturbing the main task. Note that the redundancy resolution concept of Sec. III-H is not used here but will be integrated in a future work.

Although only two major priority levels exist, a more detailed hierarchy is achieved by proper choice and design of the subtasks within one level. Moreover, the fact is exploited that several subtasks do not have any intersections. Examples are the decoupling of the upper body singularity avoidance (Sec. III-G) and the dynamic singularity avoidance of the mobile base (Sec. III-E), or the decoupling of the upper body mechanical end stop potentials and the platform impedance. Hence, undesired and undefined competitions on one priority level are avoided. See [26] for a detailed discussion of all combinations of the involved tasks.

Several experiments are conducted to demonstrate the performance of the control structure. First, the step response of the right TCP in the case of a forward motion $(\Delta x=0.2 \mathrm{~m})$ is evaluated. All subtasks are activated and the translational stiffness of the Cartesian impedance is set to $K_{\mathrm{t}}=500 \mathrm{~N} / \mathrm{m}$. As it can be seen in Fig. 14, the actual settling time is less than $0.5 \mathrm{~s}$. Besides, the overshooting is mentionable which can be traced back to the delayed behavior of the platform due to the admittance coupling, see Fig. 14 (bottom), and a damping ratio of $\zeta=0.7$ in the impedance. As the impedance is basically a PD-controller and does not possess an integrating component, a steady-state error may remain which is observable in the upper plots. The excitation in $x$-direction also affects the other two translational directions marginally. The steady-state errors can be reduced by using a higher translational stiffness.

The second experiment shows the robot behavior in the case of a continuous trajectory (see first chart in Fig. 16). The initial configuration is depicted in Fig. 15 (a). The right TCP frame is commanded to move forward $1 \mathrm{~m}$ and then back to the initial frame, see Fig. 15 (b). Apparently, the controller leads to a totally different configuration when approaching the initial frame again. The second chart in Fig. 16 depicts a quadratic
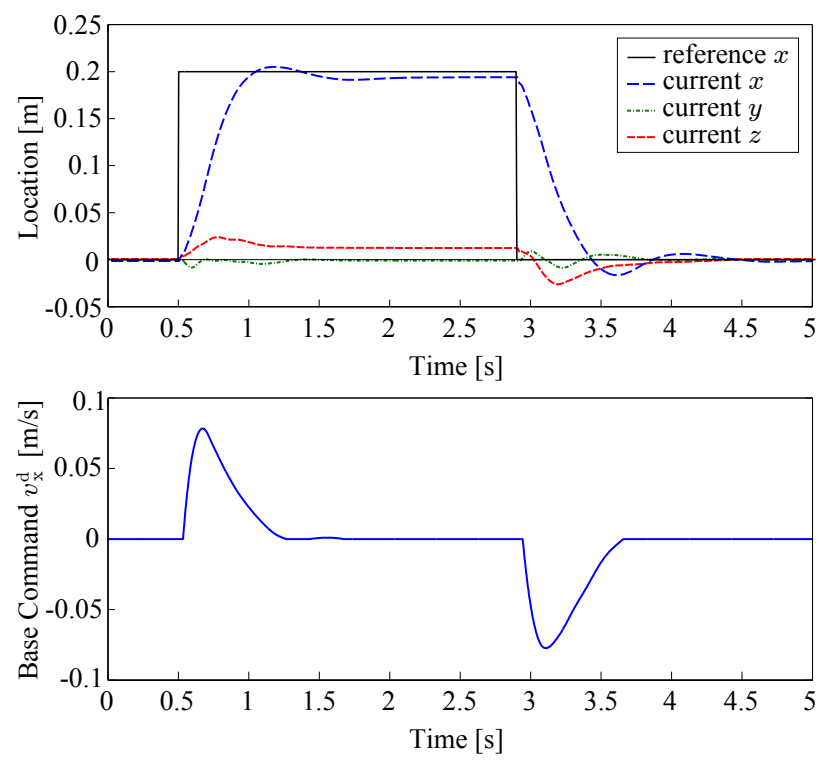

Fig. 14. Step response for a translational TCP motion in $x$-direction with a stiffness of $K_{\mathrm{t}}=500 \mathrm{~N} / \mathrm{m}$.

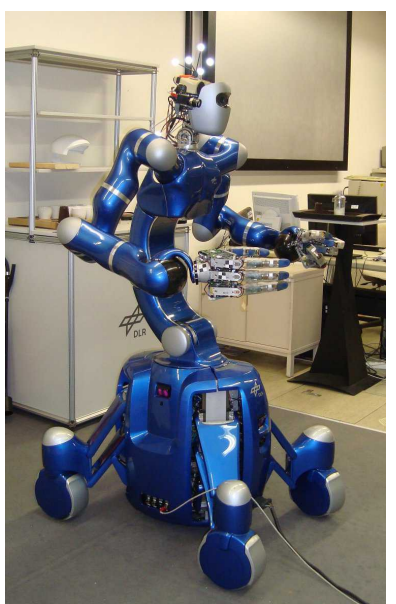

(a) $t=0 \mathrm{~s}$

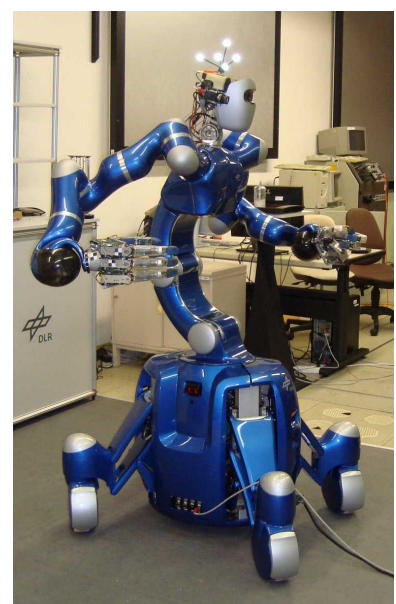

(b) $t=16 \mathrm{~s}$
Fig. 15. Snapshots during the experiment. While the right TCP is in the same configuration in both pictures, the reactive whole-body control leads to a completely different joint configuration after task execution in (b). All required controllers are computed on-board. The cables are used to facilitate the experimental evaluation.

norm of selected null space subtask torques to allow direct comparison. Obviously, returning does not lead to the same subtask participation. For example, the upper body singularity avoidance is more crucial while moving forward to prevent outstreched arms than it is when moving backward. In contrast, the avoidance of mechanical end stops is only active during the backward motion (after $11 \mathrm{~s}$ ). That complies well with the intuition of the observer when looking at the configurations of the robot (Fig. 15). Apparently, Justin is closer to its workspace boundaries in the right snapshot than it is in the left one. The third chart depicts the norms of the top priority tasks as well as the null space projection (projected subtasks from the second chart). It is noticeable that the collision avoidance only affects the behavior while moving backward. But that is actually plausible: Since the arm is faster than the inert 

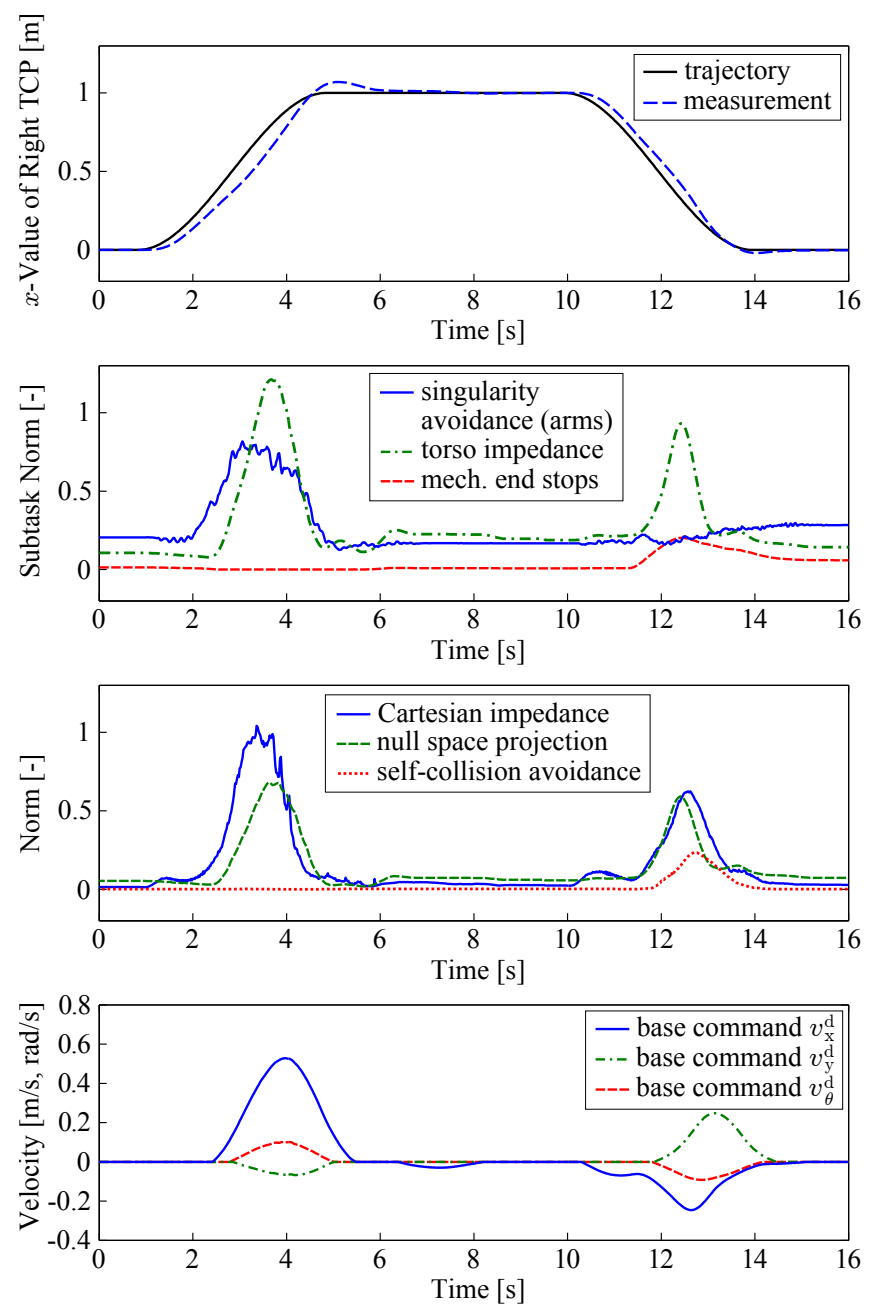

Fig. 16. Robot behavior for a continuous trajectory of the right TCP with an impedance stiffness of $K_{\mathrm{t}}=500 \mathrm{~N} / \mathrm{m}$ (translational) and $K_{\mathrm{r}}=100 \mathrm{Nm} / \mathrm{rad}$ (rotational) and a damping ratio of $\zeta=0.7$.

mobile base, a self-collision between the right hand and the torso has to be avoided while the platform is still accelerating. The last plot illustrates the base velocities which are output of the platform admittance simulation. The significantly different configuration in Fig. 15 (right) in comparison to the initial pose is primarily caused by the asymmetrical commands for the mobile platform.

The third experiment shows the performance of the controller while a human is interacting with the robot, see Fig. 17. The user pushes the right TCP away from its desired position and orientation at $t=1 \mathrm{~s}$ and $t=5 \mathrm{~s}$. Thereupon, the mobile base tries to compensate for that error (bottom plot). This, in turn, leads to a null space motion w.r.t. the Cartesian impedance task. When releasing the TCP, the remaining platform velocity and the impedance induce a small overshoot before a steady state is reached again. That effect can be reduced by applying a higher stiffness to the TCPs. Another possibility would be to consider the platform velocity within the damping design of the Cartesian impedance (5). It is also noticeable that a rotational deviation of the TCP of almost $1 \mathrm{deg}$ remains. Two possible sources can be identified: On the one hand, the missing integrating component in the impedance
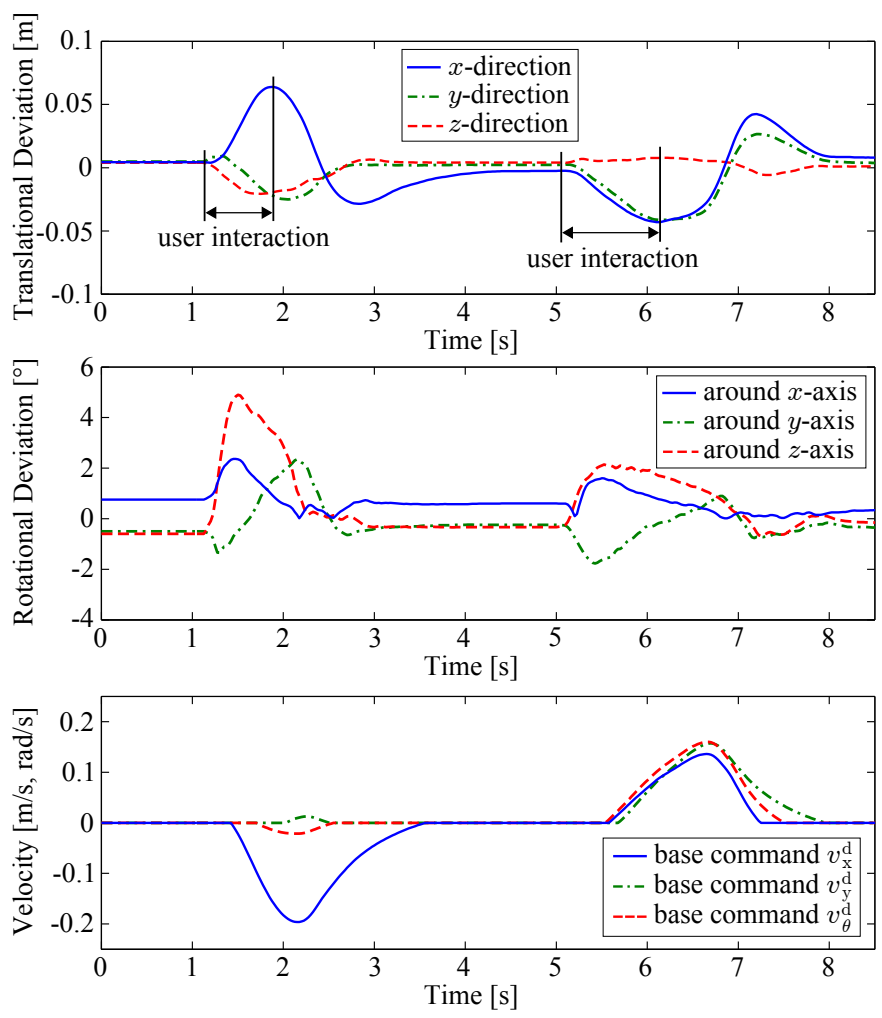

Fig. 17. TCP deviations and platform commands while interacting with a human. The robot is in reactive whole-body control mode.

controller (PD controller) prevents a zero steady-state error. On the other hand, the mobile base is designed to move only if a force threshold is exceeded. That avoids a permanent reorientation phase of the wheels in the goal configuration of the robot. Hence, even a very small intervention of the collision avoidance may cause the Cartesian impedance to miss the target slightly.

Finally, we present several snapshots from another experiment. This time an object shall be approached and grasped by the robot. In this context, an external camera tracking system is utilized. Snapshots during the motion are provided in Fig. 18. The planning is done by interpolating between the initial TCP frame and the identified object frame. The 6 DOF trajectory consists of simple 3rd order polynomials. The robot is approaching the object on the table and the platform is repelled from it when the distance is close. A naturally looking whole-body motion is achieved. Finally, Justin grasps the object and reaches the same left TCP configuration as in the beginning of the experiment. The total time amounts to about $13 \mathrm{~s}$.

\section{CONCLUSIONS}

In this article, we presented a control framework for reactive mobile manipulation of torque controlled, wheeled humanoid robots. We applied the approach to the humanoid Rollin' Justin of the DLR. A variety of simultaneous tasks was executed by utilizing the large number of DOF the manipulator is equipped with. Among other aspects, these comprise safety issues like self-collision avoidance, collision 


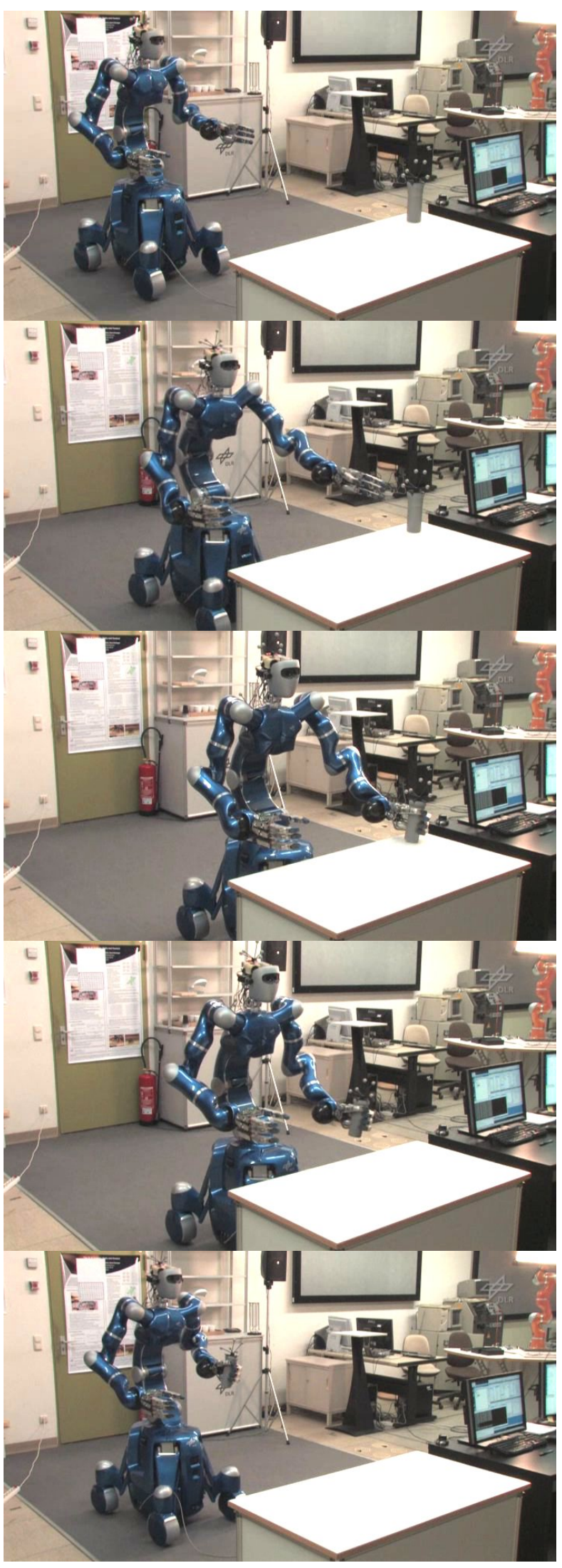

Fig. 18. The robot is grasping an object with the left hand. The 6 DOF TCP trajectory is realized while multiple objectives are reached reactively and simultaneously. avoidance with external objects and compliant interaction with the environment. Moreover, physical constraints are fulfilled and criteria like maintaining the manipulability are achieved by integrating reactive subtasks via null space projections. Robust task execution which can be planned in a low-dimensional operational space completes the framework. We integrated the newest results of the robotic community on dynamic mobile manipulation control and gave solutions to several still open questions. Various experiments on the real robotic system demonstrated the performance of our approach.

\section{REFERENCES}

[1] T. Asfour, F. Gyarfas, P. Azad, and R. Dillmann, "Imitation Learning of Dual-Arm Manipulation Tasks in Humanoid Robots," International Journal of Humanoid Robotics, vol. 5, no. 2, pp. 183-202, 2008.

[2] S. Haddadin, A. Albu-Schäffer, and G. Hirzinger, "Requirements for Safe Robots: Measurements, Analysis and New Insights," International Journal of Robotics Research, vol. 28, no. 11-12, pp. 1507-1527, Nov./Dec. 2009.

[3] Y. Sakagami, R. Watanabe, C. Aoyama, S. Matsunaga, N. Higaki, and K. Fujimura, "The intelligent ASIMO: System overview and integration," in Proc. of the 2002 IEEE/RSJ International Conference on Intelligent Robots and Systems, October 2002, pp. 2478-2483.

[4] M. Diftler, J. Mehling, M. Abdallah, N. Radford, L. Bridgwater, A. Sanders, R. Askew, D. Linn, J. Yamokoski, F. Permenter, B. Hargrave, R. Platt, R. Savely, and R. Ambrose, "Robonaut 2 - The First Humanoid Robot in Space," in Proc. of the 2011 IEEE International Conference on Robotics and Automation, May 2011, pp. 2178-2183.

[5] K. Kaneko, F. Kanehiro, S. Kajita, H. Hirukawa, T. Kawasaki, M. Hirata, K. Akachi, and T. Isozumi, "Humanoid Robot HRP-2," in Proc. of the 2004 IEEE International Conference on Robotics and Automation, April 2004, pp. 1083-1090.

[6] C. Borst, T. Wimböck, F. Schmidt, M. Fuchs, B. Brunner, F. Zacharias, P. R. Giordano, R. Konietschke, W. Sepp, S. Fuchs, C. Rink, A. AlbuSchäffer, and G. Hirzinger, "Rollin' Justin - Mobile Platform with Variable Base," in Proc. of the 2009 IEEE International Conference on Robotics and Automation, May 2009, pp. 1597-1598.

[7] T. Asfour, K. Regenstein, J. Schröder, A. Bierbaum, N. Vahrenkamp, and R. Dillmann, "ARMAR-III: An Integrated Humanoid Platform for Sensory-Motor Control," in Proc. of the 6th IEEE-RAS International Conference on Humanoid Robots, December 2006, pp. 169-175.

[8] H. Iwata and S. Sugano, "Design of Human Symbiotic Robot TWENDYONE," in Proc. of the 2009 IEEE International Conference on Robotics and Automation, May 2009, pp. 580-586.

[9] “PR2," Willow Garage, http://www.willowgarage.com/pr2, Jan. 2012.

[10] O. Khatib, "A Unified Approach for Motion and Force Control of Robot Manipulators: The Operational Space Formulation," IEEE Journal of Robotics and Automation, vol. RA-3, no. 1, pp. 43-53, February 1987.

[11] L. Sentis and O. Khatib, "Synthesis of Whole-Body Behaviors through Hierarchical Control of Behavioral Primitives," International Journal of Humanoid Robotics, vol. 2, no. 4, pp. 505-518, January 2005.

[12] K. Nagasaka, Y. Kawanami, S. Shimizu, T. Kito, T. Tsuboi, A. Miyamoto, T. Fukushima, and H. Shimomura, "Whole-body Cooperative Force Control for a Two-Armed and Two-Wheeled Mobile Robot Using Generalized Inverse Dynamics and Idealized Joint Units," in Proc. of the 2010 IEEE International Conference on Robotics and Automation, May 2010, pp. 3377-3383.

[13] M. Behnisch, R. Haschke, and M. Gienger, "Task Space Motion Planning Using Reactive Control," in Proc. of the 2010 IEEE/RSJ International Conference on Intelligent Robots and Systems, October 2010, pp. 5934-5940.

[14] O. Khatib, L. Sentis, J. Park, and J. Warren, "Whole-Body Dynamic Behavior and Control of Human-like Robots," International Journal of Humanoid Robots, vol. 1, no. 1, pp. 29-43, March 2004.

[15] O. Brock and O. Khatib, "Elastic Strips: A Framework for Motion Generation in Human Environments," International Journal of Robotics Research, vol. 21, no. 12, pp. 1031-1052, December 2002.

[16] O. Khatib, "Real-Time Obstacle Avoidance for Manipulators and Mobile Robots," International Journal of Robotics Research, vol. 5, no. 1, pp. 90-98, Spring 1986. 
[17] B. Siciliano and J.-J. Slotine, "A General Framework for Managing Multiple Tasks in Highly Redundant Robotic Systems," in Proc. of the 5th International Conference on Advanced Robotics, June 1991, pp. 1211-1216.

[18] O. Brock, O. Khatib, and S. Viji, "Task-Consistent Obstacle Avoidance and Motion Behavior for Mobile Manipulation," in Proc. of the 2002 IEEE International Conference on Robotics and Automation, May 2002. pp. 388-393.

[19] N. Mansard, O. Khatib, and A. Kheddar, "A Unified Approach to Integrate Unilateral Constraints in the Stack of Tasks," IEEE Transactions on Robotics, vol. 25, no. 3, pp. 670-685, June 2009.

[20] M. Stilman, "Global Manipulation Planning in Robot Joint Space With Task Constraints," IEEE Transactions on Robotics, vol. 26, no. 3, pp. 576-584, June 2010.

[21] D. Berenson, S. Srinivasa, and J. Kuffner, "Task Space Regions: A Framework for Pose-Constrained Manipulation Planning," International Journal of Robotics Research, vol. 30, no. 12, pp. 1435-1460, October 2011.

[22] A. Dietrich, T. Wimböck, H. Täubig, A. Albu-Schäffer, and G. Hirzinger, "Extensions to Reactive Self-Collision Avoidance for Torque and Position Controlled Humanoids," in Proc. of the 2011 IEEE International Conference on Robotics and Automation, May 2011, pp. 3455-3462.

[23] A. Dietrich, T. Wimböck, A. Albu-Schäffer, and G. Hirzinger, "Singularity Avoidance for Nonholonomic, Omnidirectional Wheeled Mobile Platforms with Variable Footprint," in Proc. of the 2011 IEEE International Conference on Robotics and Automation, May 2011, pp. 61366142.

[24] A. Dietrich, A. Albu-Schäffer, and G. Hirzinger, "On Continuous Null Space Projections for Torque-Based, Hierarchical, Multi-Objective Manipulation," in Proc. of the 2012 IEEE International Conference on Robotics and Automation, May 2012, pp. 2978-2985.

[25] P. R. Giordano, M. Fuchs, A. Albu-Schäffer, and G. Hirzinger, "On the Kinematic Modeling and Control of a Mobile Platform Equipped with Steering Wheels and Movable Legs," in Proc. of the 2009 IEEE International Conference on Robotics and Automation, May 2009, pp. 4080-4087.

[26] A. Dietrich, T. Wimböck, and A. Albu-Schäffer, "Dynamic Whole-Body Mobile Manipulation with a Torque Controlled Humanoid Robot via Impedance Control Laws," in Proc. of the 2011 IEEE/RSJ International Conference on Intelligent Robots and Systems, September 2011, pp. 3199-3206.

[27] A. Albu-Schäffer, C. Ott, and G. Hirzinger, "A Unified Passivity-based Control Framework for Position, Torque and Impedance Control of Flexible Joint Robots," International Journal of Robotics Research, vol. 27, no. 1, pp. 23-39, January 2007.

[28] E. G. Gilbert, D. W. Johnson, and S. S. Keerthi, "A Fast Procedure for Computing the Distance Between Complex Objects in ThreeDimensional Space," IEEE Journal of Robotics and Automation, vol. 4, no. 2, pp. 193-203, April 1988

[29] A. Albu-Schäffer, C. Ott, U. Frese, and G. Hirzinger, "Cartesian Impedance Control of Redundant Robots: Recent Results with the DLR-Light-Weight-Arms," in Proc. of the 2003 IEEE International Conference on Robotics and Automation, Sept. 2003, pp. 3704-3709.

[30] B. Thuilot, B. D'Andréa-Novel, and A. Micaelli, "Modeling and Feedback Control of Mobile Robots Equipped with Several Steering Wheels," IEEE Transactions on Robotics and Automation, vol. 12, no. 3, pp. 375390, June 1996

[31] C. P. Connette, C. Parlitz, M. Hägele, and A. Verl, "Singularity Avoidance for Over-Actuated, Pseudo-Omnidirectional, Wheeled Mobile Robots," in Proc. of the 2009 IEEE International Conference on Robotics and Automation, May 2009, pp. 4124-4130.

[32] C. Ott, Cartesian Impedance Control of Redundant and Flexible-Joint Robots, ser. Springer Tracts in Advanced Robotics. Springer Publishing Company, Berlin Heidelberg, 2008, vol. 49.

[33] A. Dietrich, T. Wimböck, A. Albu-Schäffer, and G. Hirzinger, "Integration of Reactive, Torque-Based Self-Collision Avoidance Into a Task Hierarchy," IEEE Transactions on Robotics, vol. 28, no. 6, pp. 12781293, December 2012.

[34] T. Kröger, "Online Trajectory Generation: Straight-Line Trajectories," IEEE Transactions on Robotics, vol. 27, no. 5, pp. 1010-1016, October 2011.

[35] R. Haschke, E. Weitnauer, and H. Ritter, "On-Line Planning of TimeOptimal, Jerk-Limited Trajectories," in Proc. of the 2008 IEEE/RSJ International Conference on Intelligent Robots and Systems, September 2008, pp. 3248-3253.

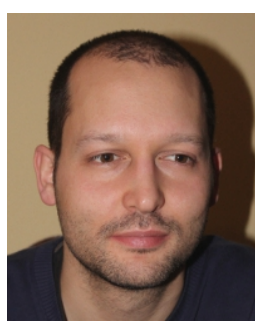

Alexander Dietrich received his Dipl.-Ing. degree in mechanical engineering with focus on control theory and fundamentals in engineering sciences from the Technical University of Munich (TUM) in 2008. In 2010, he joined the German Aerospace Center (DLR), Institute of Robotics and Mechatronics, as a research scientist. His current research interests include impedance and force control, whole-body mobile manipulation, kinematic redundancy, and safe physical human-robot interaction.

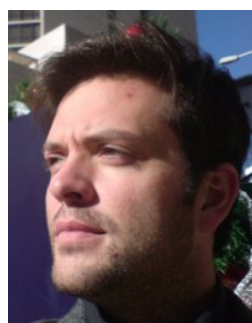

Thomas Wimböck studied electrical engineering at the Rensselaer Polytechnic Institute, Troy, and at the Ecole Polytechnique Fédérale de Lausanne, Switzerland. He received his B.Sc and M.Sc. (in 2004) degrees in electrical engineering from TUM In 2004, he joined the DLR, Institute of Robotics and Mechatronics, as a research scientist. His main research interests include nonlinear control, dexterous robot hands, impedance control, VS control, twohanded (humanoid) manipulation, and kinematic redundancy.

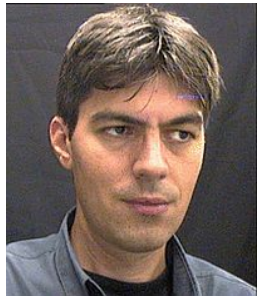

Alin Albu-Schäffer received the Dipl.-Ing. degree in electrical engineering from the Technical University of Timisoara, Romania in 1993 and the Ph.D. degree in control systems from TUM in 2002. Since 1995, he has been working with the DLR, Institute of Robotics and Mechatronics, Germany, where he is currently head of the department for mechatronic components and systems. His current research interests include robot modeling and control, nonlinear control, flexible joint robots, impedance and force able impedance actuation. control, physical human-robot interaction, and vari-

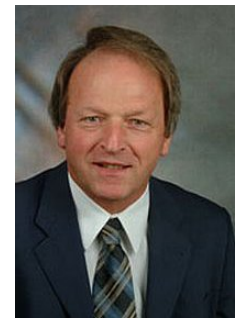

Gerd Hirzinger received the Dipl.-Ing. and Ph.D. degrees from TUM, Germany, in 1969 and 1974, respectively. Since 1992, he has been the director at the DLR, Institute of Robotics and Mechatronics, Wessling, Germany. In 1991, he received a joint professorship from TUM and in 2003, an honorary professorship at the Harbin Institute of Technology, China. He was prime investigator of the first remote control space robot ROTEX, which flew onboard the shuttle Columbia in April 1993. He is the recipient of numerous national and international awards, including the Joseph-Engelsberger-Award for achievements in robotic science in 1994, the Leibniz-Award, in 1995, the Japan Robotics Association (JARA) Award, the Karl-Heinz-Beckurts-Award in 1996, and the IEEE Fellow Award in 1997. In 2004, he received the order of merit of the Federal Republic of Germany and became a member of the Wall of Fame of the Heinz Nixdorf Computer Museum. He is also the recipient of the IEEE Pioneer Award of the Robotics and Automation Society, the 2005 Honorary Citizenship of Budapest Tech, and the IEEE Field Award Robotics and Automation in 2007. 